\title{
EchoGéo
}

4 | 2008

Archéologie et Géographie

\section{La forteresse de Sadr, une lecture géo-historique au temps des croisés}

Corinne Feïss-Jehel, Jean-Michel Mouton, Claudine Piaton, Pierre-Jérôme Jehel et Jeannine Le Rhun

\section{OpenEdition}

\section{Journals}

Édition électronique

URL : https://journals.openedition.org/echogeo/2228

DOI : $10.4000 /$ echogeo.2228

ISSN : 1963-1197

Éditeur

Pôle de recherche pour l'organisation et la diffusion de l'information géographique (CNRS UMR 8586)

Référence électronique

Corinne Feïss-Jehel, Jean-Michel Mouton, Claudine Piaton, Pierre-Jérôme Jehel et Jeannine Le Rhun, "La forteresse de Sadr, une lecture géo-historique au temps des croisés », EchoGéo [En ligne], 4 | 2008, mis en ligne le 27 octobre 2011, consulté le 31 juillet 2021. URL : http://journals.openedition.org/ echogeo/2228; DOI : https://doi.org/10.4000/echogeo.2228

Ce document a été généré automatiquement le 31 juillet 2021.

EchoGéo est mis à disposition selon les termes de la licence Creative Commons Attribution - Pas d'Utilisation Commerciale - Pas de Modification 4.0 International (CC BY-NC-ND) 


\title{
La forteresse de Sadr, une lecture géo-historique au temps des croisés
}

\author{
Corinne Feïss-Jehel, Jean-Michel Mouton, Claudine Piaton, Pierre-Jérôme \\ Jehel et Jeannine Le Rhun
}

\section{Présentation et intérêt du site}

1 Au coeur du désert du Sinaï, la forteresse de Sadr, appelée aujourd'hui Qal-at al-Gindi, constitue le témoignage archéologique le plus authentique laissé au Proche-Orient par le célèbre souverain kurde Saladin (1174-1193). Cet édifice construit dans le contexte des croisades est en effet un des rares exemples de l'architecture militaire musulmane des XIIe-XIIIe siècles préservé dans son état d'origine (Figure 0). La plupart des monuments de ce type, que l'on trouve notamment en Syrie, ont été occupés sur une longue période, ont été maintes fois remaniés et sont bien souvent passés des mains des croisés à celles des musulmans sans que l'on sache vraiment la date et l'ampleur des remaniements que l'on doit attribuer à chacun des deux partis. L'intérêt de la forteresse de Sadr tient précisément au fait qu'elle ne soit jamais passée aux mains des croisés bien qu'elle fût une fois assiégée en 1177. De plus, la faible durée d'occupation moins d'un siècle - et son isolement font de ce site un milieu clos, certes très ruiné, mais où l'ensemble du matériel est resté sur place, n'a pas été pillé et peut permettre une anastylose quasi complète. Elle est l'un des sites proposés par l'Egypte pour une inscription sur la liste du patrimoine mondial de l'UNESCO.Le caractère exceptionnel de ce site vient aussi de sa situation géographique très particulière sur un éperon rocheux dominant de près de 150 mètres les étendues planes et arides du désert de Tih. 


\section{Le contexte historique ou Pourquoi bâtir une forteresse au milieu du désert et pourquoi ce fut possible?}

2 La forteresse de Sadr a été construite au début des années 1170 par le célèbre sultan kurde Saladin. Celui-ci venait l'année précédente de faire la conquête de l'Egypte avec son oncle Shîrkûh pour le compte du prince turc de Syrie, Nûr al-Dîn. Dans les mois qui suivirent cette conquête, Saladin devint vizir d'Egypte pour le compte du dernier calife fatimide et commença ainsi sa remarquable carrière politique et militaire. En effet, dès l'année 1171 il renversa le calife fatimide d'Egypte et en 1174, il s'empara de Damas, capitale de la Syrie, à la mort de son maître Nûr al-Dîn dont il s'appropria les terres. La construction de la forteresse de Sadr vers 1175 s'inscrit ainsi dans un contexte d'unification politique de la Syrie musulmane et de l'Egypte sous l'autorité de Saladin. Cette forteresse devait commander la route du Sinaï central, appelée route de Sadr à Ayla, axe majeur qui permettait de relier les deux capitales de l'Empire ayyoubide, Le Caire et Damas, tout en contournant les Etats croisés fondés sur la côte méditerranéenne depuis 1099. La position dominante du site de Sadr et la présence d'un point d'eau dans ce désert hostile donnaient à cette forteresse une valeur stratégique et en faisaient le passage obligé de Saladin et de ses armées se rendant d'Egypte en Syrie. Cette position stratégique disparut subitement en 1187 après Hattîn et la chute de Jérusalem; en effet, après avoir achevé la conquête des principautés musulmanes de Syrie du Nord (Alep en 1183) et de Haute Mésopotamie, Saladin entreprit la guerre sainte ou jihâd contre les croisés. Sa principale cible fut le Royaume latin de Jérusalem qui s'effondra en cette année 1187 permettant la réouverture de la route du Nord-Sinaï, beaucoup plus courte, pour les communications entre Le Caire et Damas. Sadr fut, semble-t-il, alors abandonnée jusqu'à la fin du règne de Saladin qui mourut en 1193, puis plus ou moins ruinée par les tremblements de terre de 1202 et 1212. Cependant la forteresse connut une seconde vie à partir de 1221 sous le règne du neveu de Saladin, l'Ayyoubide al-Malik al-Kâmil, qui la fit restaurer au moment où il s'apprêtait à livrer Jérusalem aux Croisés. Les dernières traces d'occupation trouvées sur le site datent du règne du dernier prince ayyoubide al-Sâlih Najm al-Dîn Ayyûb qui en fit une prison pour y enfermer les mamlouks de son père. Cette phase d'abandon, au milieu du XIIIe siècle, correspond aussi au retour définitif de Jérusalem dans le giron de l'islam. 
Figure 0

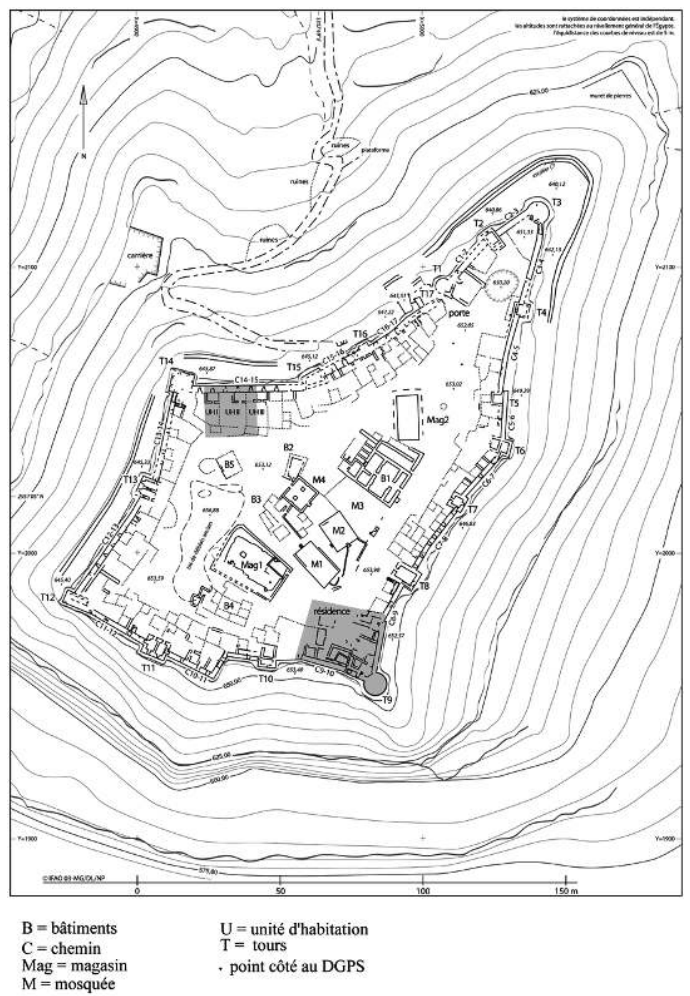

3 L'organisation des traversées est-ouest du Sinaï a connu un bouleversement majeur dans le courant du XIIe siècle.

4 La route côtière basse, qui longeait la Méditerranée, permettait un chemin aisé et rapide pour les caravanes musulmanes qui effectuaient la liaison Egypte-Syrie. La plaine littorale du Gifâr offrait en effet un secteur dunaire relativement plat eu égard aux massifs plateaux et montagnes du Sinaï central. Cette route qui fut la route principale pendant des siècles se trouva pour partie sous le contrôle des Etats francs en 1099 avec la création de Royaume latin de Jérusalem.

5 L'emprise latine devient un obstacle plus infranchissable que les barrières physiques du Sinaï. Saladin met en place une route intérieure, moins rapide, plus délicate mais sûre pour relier l'Afrique à l'Asie musulmane. Le contrôle des contraintes physiques devient peu à peu un allié contre les croisés. L'emplacement de la forteresse de Sadr semble répondre à deux critères majeurs. Le site a été choisi, d'une part, pour son caractère défensif. Campée sur haute butte témoin, la forteresse bénéficie d'un commandement de choix sur les secteurs bas, seuls voies de passage possible pour une armée et d'autre part il est situé dans un secteur très particulier et privilégié en ce qui concerne les possibilités d'alimentation en eau douce La forteresse de Sadr dans le centre Sinaï, à de $80 \mathrm{~km}$ à l'est du golfe de Suez, sur la vallée du Wâdi-al-Sudr. a été bâtie sur et pour la route du Sinaï centrale. A mi chemin entre le Caire et la forteresse d'Ayla, elle a constitué une halte vitale pour Saladin dans un secteur extrêmement aride et austère 


\section{Géomorphologie du Sinaï Nord et son utilisation au XII ème siècle en pleine période des croisades}

6 L'Afrique du Nord-Est est constituée par un fragment de vieux socle pré-cambrien qui a subit les cycles d'érosion du primaire. La couverture sédimentaire débute avec des dépôts carbonifêres mais c'est avec les grès nubiens, épaisse masse de sédiments continentaux du secondaire que la couverture se réalise vraiment (Figure1). Ces grès nubiens surmontent d'épaisses séries crétacé (marine) et nummulitique ${ }^{1}$.

Figure 1

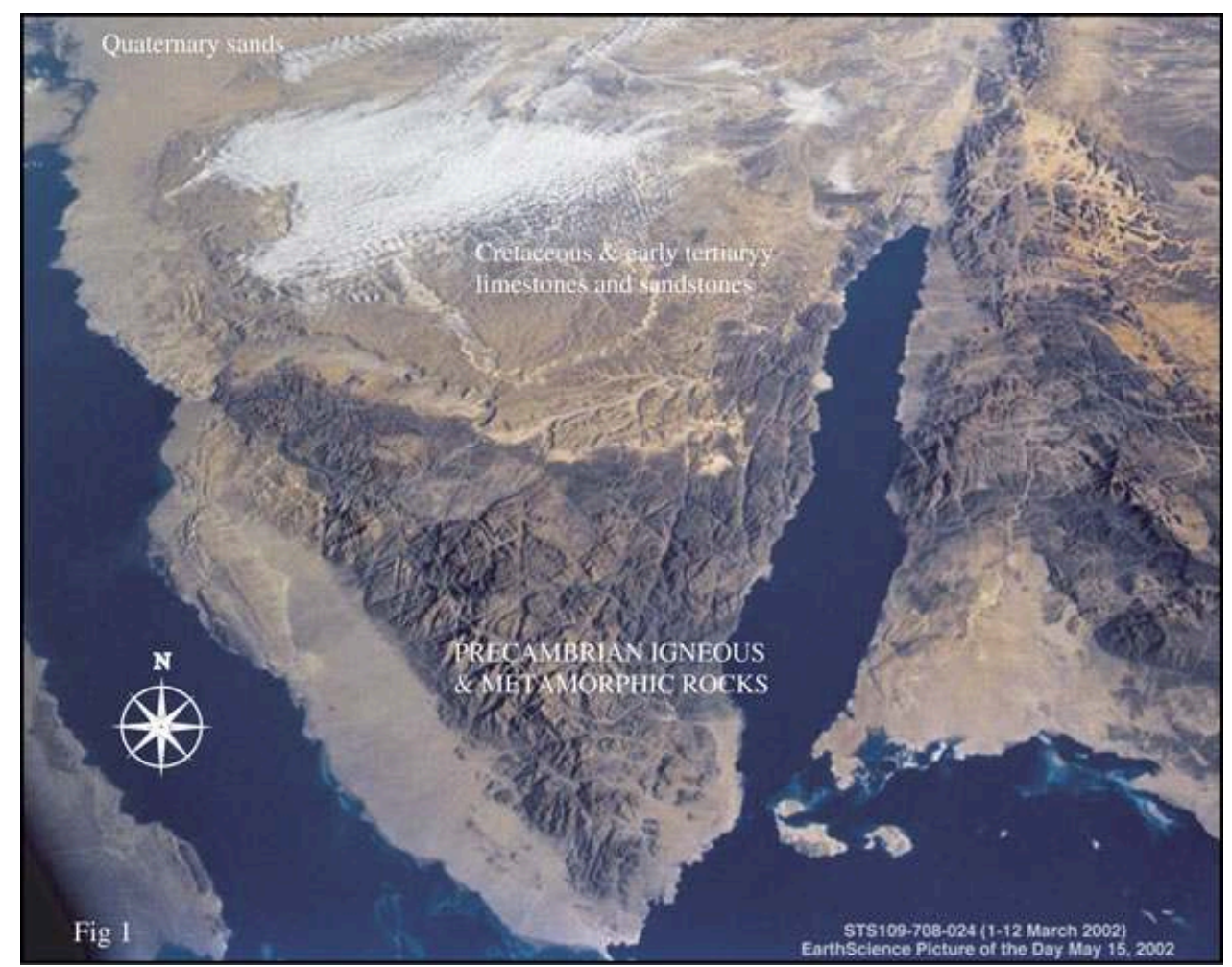

7 La péninsule du Sinaï correspond à un horst de forme triangulaire basculé vers le Nord (Figure 2). 


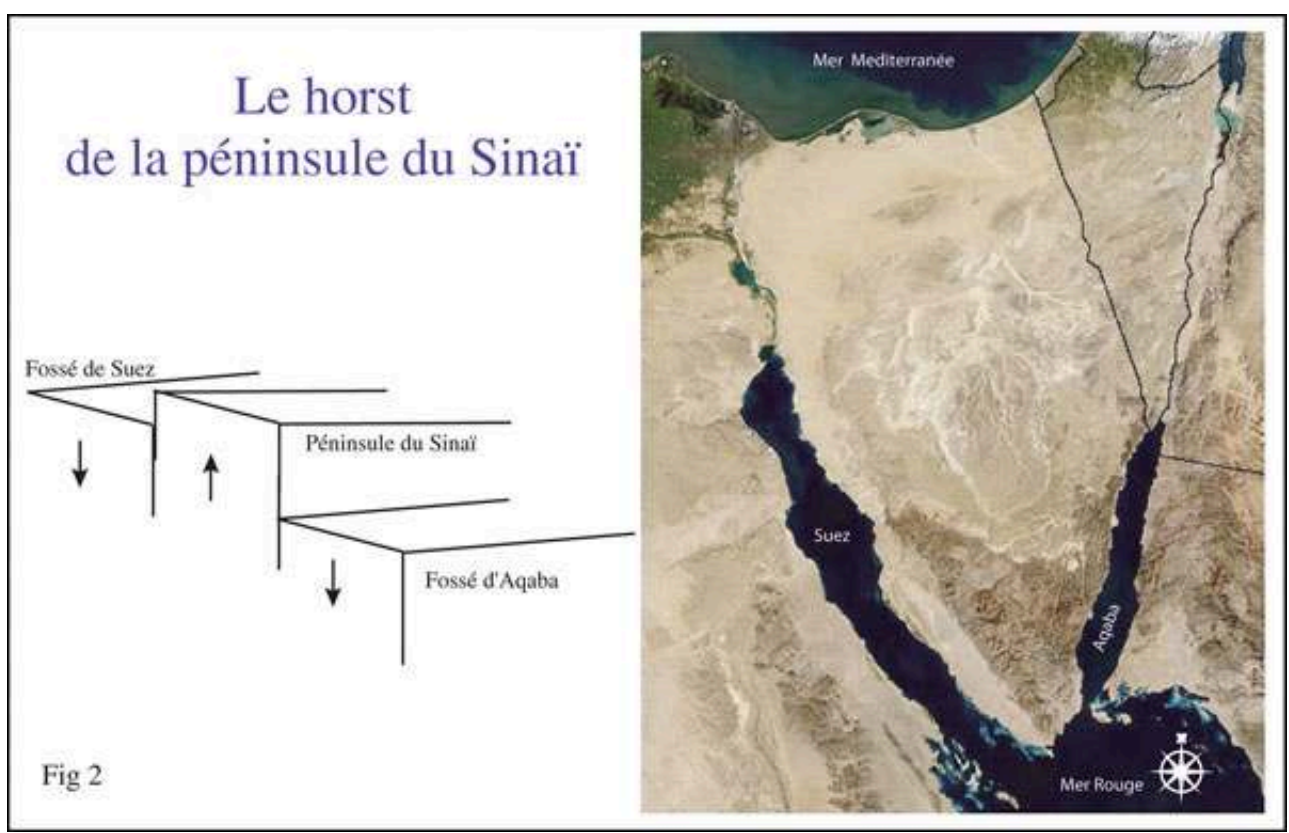

Cette situation tectonique va favoriser le développement de la côte méditerranéenne par la présence d'une large plaine littorale.

9 A la période qui nous intéresse, ce secteur va permettre le développement de villes et d'une route côtière bordant la méditerranée et reliant l'Egypte au Royaume de Jérusalem. C'est précisément le contrôle de cette route par les états croisés qui va engendrer la nécessité d'une route intérieure permettant aux caravanes et aux cavaliers de Saladin de rejoindre la Terre Sainte.

Suez et Aqaba, fossés tectoniques et fossés religieux

Le horst est limité par les fossés de Suez et Aqaba. Tandis que l'escarpement oriental plonge directement dans la mer, l'escarpement occidental qui descend obliquement par rapport au rivage constitue un flanc haché de cassures et de compartiments dénivelés en escalier qui s'incline vers le golfe d'Aqaba (1000 $\mathrm{m}$ de profondeur). Ce jeu de cassure va permettre l'installation d'ourlets sableux le long de la côte.

A l'ouest, le fossé de Suez plus large mais nettement moins profond $(100 \mathrm{~m})$ se trouve bordé par une plaine littorale d'environ $8 \mathrm{~km}$ que l'on ne retrouve pas sur la façade orientale.

Ces deux golfes, passages obligés, entre Egypte et Syrie vont devenir les théâtres de conflits et d'enjeux stratégiques.

La seigneurie d'Outre-Jourdain (1177) fut confiée par le roi Baudouin IV à Renaud de Chatillon. Ce secteur franc contrôlait la route du Hadj empruntée par les pèlerins allant à la Mecque. C'est pourquoi les forteresses de Ailat et de Jazîrat Fir'awun sur l'île de Graye à $15 \mathrm{~km}$ au sud-ouest d'Ailat vont représenter un intérêt tout particulier ${ }^{2}$. Sous le fossé de la mer Rouge se trouvent en effet des blocs allongés dans le sens du fossé, qui portent des îles volcaniques.

Les riches caravanes du Hadj payaient certes un tribut mais elles laissaient présager de fortunes encore bien plus grandes dans les villes saintes. Renaud de Châtillon pensa reconquérir le fortin de Graye (Jazîrat - Fir'awun) dans le golfe d'Aqaba, qui était tombé 
entre les mains de Saladin en 1175, et ce afin de s'assurer de la suprématie maritime ${ }^{3}$. Il décida de construire des navires à Ascalon et Montréal et de les acheminer par chameaux non loin des plages d'Aïlat ${ }^{4}$. Les Francs considéraient cette entreprise comme une double opportunité. Elle représentait un enjeu commercial et politique. Il s'agissait de contrôler l'une des grandes artères commerciales de l'lslam. Les côtes d'Afrique, de l'Inde et de l'Extrême-Orient expédiaient en effet leurs marchandises, par Aden et le Bab el-Mandeb ${ }^{5}$, aux ports de l'Est de l'Egypte et de maitriser toutes les routes du Hadj puisque de l'Afrique du Nord, depuis le Maroc jusqu'à l'Egypte, elles aboutissaient à la côte orientale égyptienne.

17 Caravanes et cavaliers du djihâd faisaient route par la voie de terre intérieure qui avait été mise au point et sécurisée par Saladin, de Jisr Al Qûlzûm, Sadr et sa forteresse, et Aqaba.

Les nouvelles de l'expédition de Renaud de Châtillon en mer Rouge étaient parvenues jusqu'au Caire. Saladin fit démonter puis acheminer par chameaux des bâtiments de guerre du port de Damiette à la marine de Qulzum, dans le golfe de Suez ${ }^{6}$. En 1183, la flotte pénétra dans le golfe d'Aqaba, la forteresse insulaire de l'île de Graye fut reprise après une bataille entre les navires égyptiens et les galères franques ${ }^{7}$. La campagne en mer Rouge permit à Saladin de recouvrer la route entre l'Egypte et la Terre Sainte.

19 Vers le Nord le socle plonge sous la couverture sédimentaire. Le paysage est celui de grandes cuestas. Puis la région des dômes structure l'espace par des alignements O.S.O./ E. N.E , avant pays de la plate forme arabique. Le Sinaï est ainsi isolé, compartimenté par deux fossés tectoniques.

Le premier relief d'importance depuis la région septentrionale des dunes littorales du Nord, est celui du Jabal Maghara, dont les altitudes varient de 500 à $750 \mathrm{~m}$. Il s'agit d'une structure nettement anticlinale qui constitue une première ride dissymétrique. Le Jabal Hamay, le jabal Umm-Asagil et le Jabal Umm-Mafrout constituent une série de dômes secondaires, répliques anticlinales du dôme de Maghara.

21 Plus au sud les Jabal Yelleq à l'ouest de la péninsule et al-Hallal à l'Est répercutent les axes O.S.O et E.N.E. de ces plissements.

22 Le Jabal al-Gidi et Râhâ prolongent vers le Sud l'alignement Yelleq / Hallal par une crête massive et clairement dessinée. C'est sur ces dernières structures que Saladin va s'appuyer pour bâtir sa forteresse. Cette Dernière ligne d'écho des plissements va servir d'ancrage pour la forteresse de Sadr qui constitue l'étape vitale de la route entre l'Egypte et la Terre Sainte.

\section{La forteresse de Sadr, une position structurale et géopolitique.}

23 Dans un contexte où les Etats croisés menacent l'islam, contrôlent Jérusalem, la maîtrise d'une route capable de relier les deux parties de son empire, la Syrie et l'Egypte sera une question vitale pour Saladin.

24 Le Sinaï central devient un enjeu stratégique majeur alors que les Etats latins condamnent les routes du littoral. Ces derniers étaient en effet assez puissants pour contrôler la route côtière basse qui borde la Méditerranée dans le nord Sinaï barrant ainsi l'accès le plus aisé aux caravanes et aux armées musulmanes et étendre le 
Royaume de Jérusalem par le sud ancrant ainsi l'emprise des croisés sur les côtes de la mer Rouge.

Trouver une nouvelle route pour relier Le Caire à la Syrie via le Sinaï et défendre cet espace contre les croisés s'impose donc à Saladin. La route intérieure Jisr Al Qûlzûm, Sadr et Aqaba emprunte le tracé de la vallée de Sudr. Cette vallée présente une entaille, entre de hauts plateaux inhospitaliers et aux franchissements délicats, assez large tout le long de son cours pour permettre le passage de cavaliers et de caravanes (Figure 3) La forteresse est bâti à l'amont du Sudr sur une haute butte témoin qui domine son cours.

Figure 3

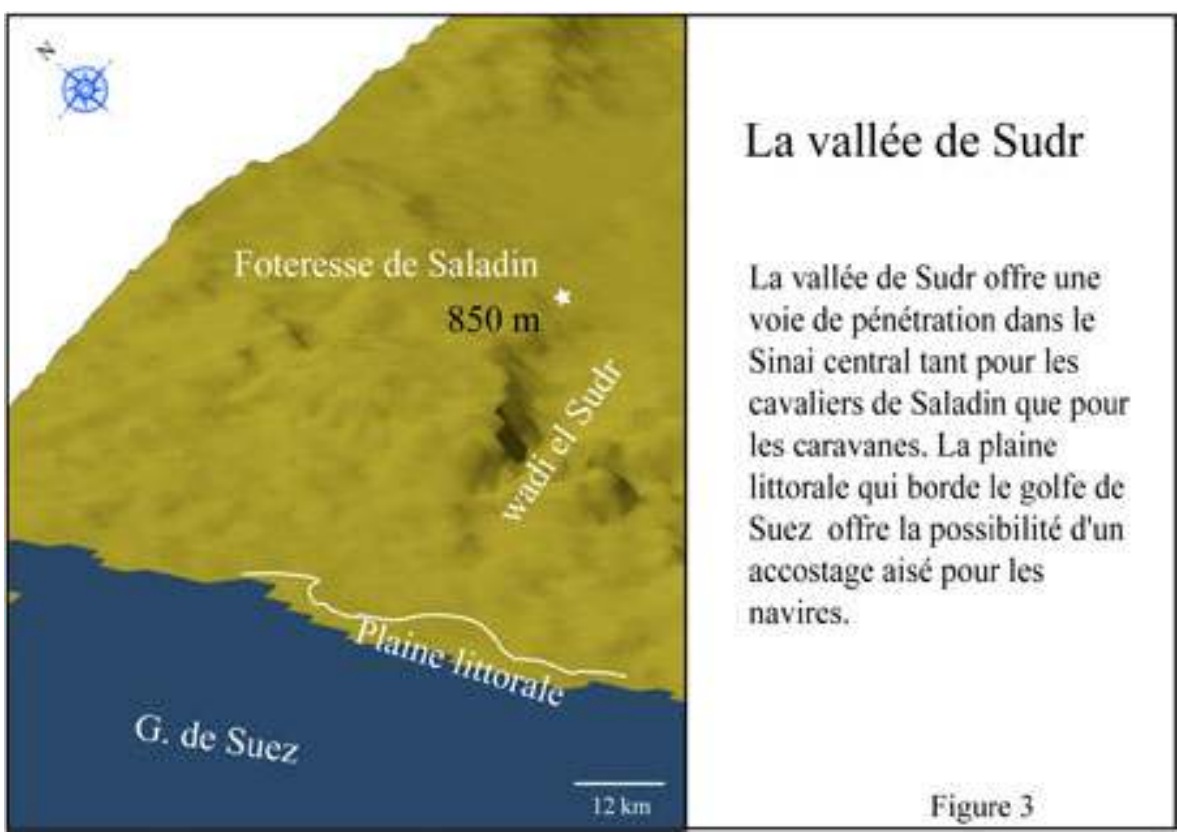

La forteresse est en effet située dans un imposant secteur de hauts plateaux dont la régularité n'exclut pas de petites ondulations calées sur les mêmes axes tectoniques que ceux des dômes septentrionaux de la péninsule. Elle constitue un bastion avancé du Jabal Râhâ dans le prolongement méridional du Jabal.al- Gidi (Figure 4). 
Figure 4

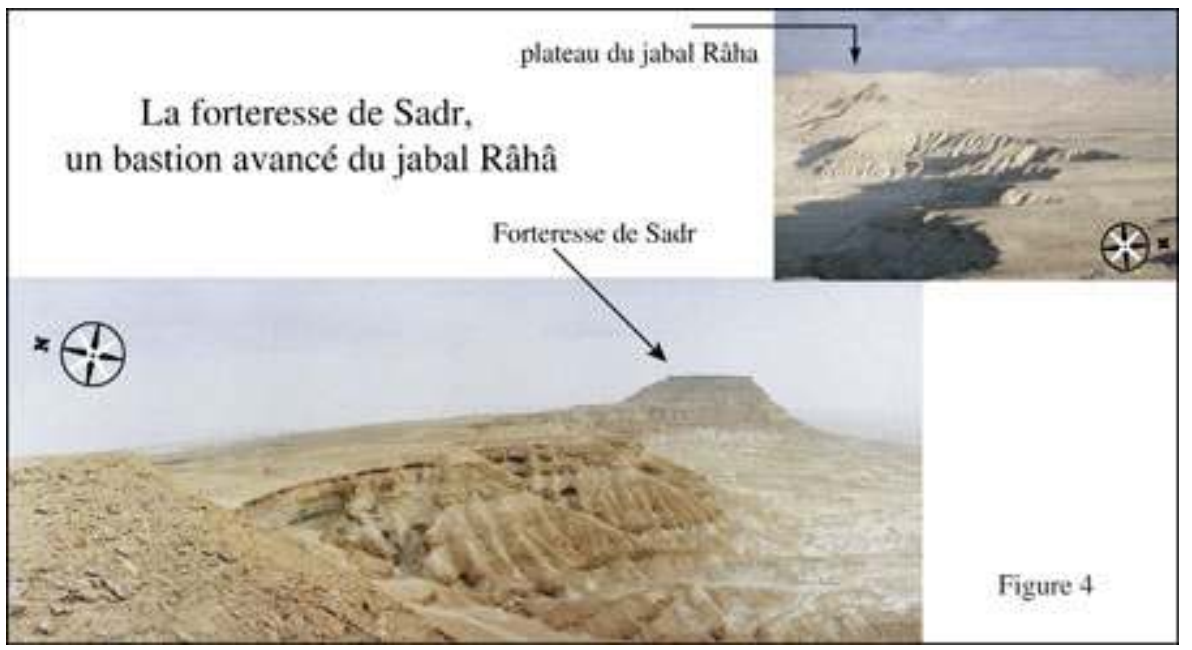

Dans ce secteur la craie du Maestrichien et du Campanien tapisse les secteurs bas, tandis que les hauts plateaux sont couronnés par une carapace de calcaires nummulitiques. C'est la présence de ces craies assez peu résistantes qui ont permis un développement de la vallée de Sudr relativement important eu égard le secteur. (figure 5).

Figure 5

Schéma des composantes géologiques du golfe de Suez vers le D. Sumar

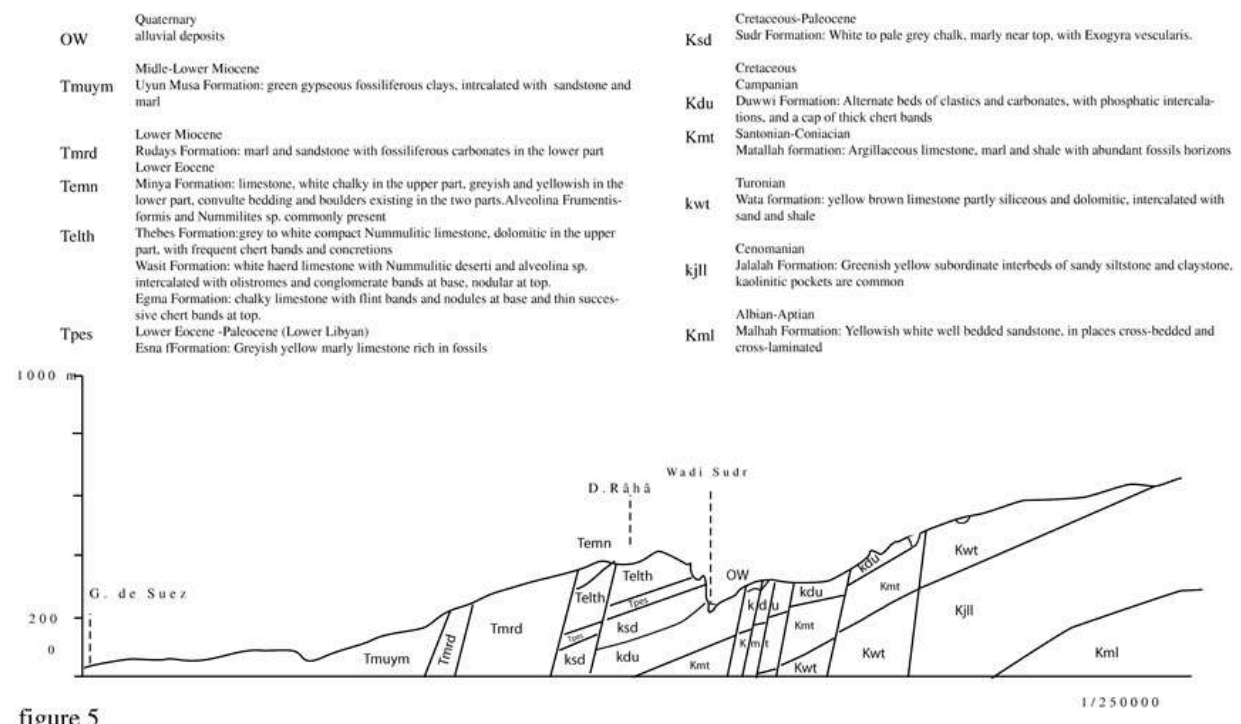

La butte est couronnée par les calcaires du Libyen inférieure. Ses flancs présentent un paysage de badlands développé dans les craies blanche de la formation de Sudr. (figure. 6) 
Les composantes géologiques de la forterese de Sadr
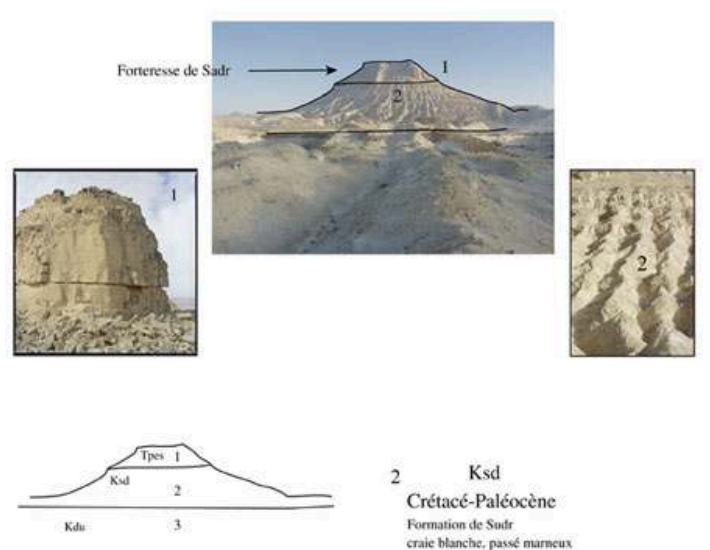
Formation de Suatr

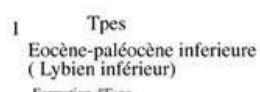
3 Krétacé Crétacé Campanien
Formation de Duwwi:

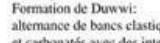
Kmt Santonien-Coniacie Formation de Matallah: Figure 6

A l'Est, les plateaux nummulitiques (Jabal Râhâ, Jabal Bodhia) et crétacé (Jabal Somar) se raccordent à un imposant glacis qui correspond à une zone d'épandage où les oueds s'étendent sans entrave et s'orientent vers la Méditerranée. Le Wâdî al-'Arîsh constitue l'axe majeur du drainage de ce vaste plan incliné vers le Nord (Figure 7).

Figure 7

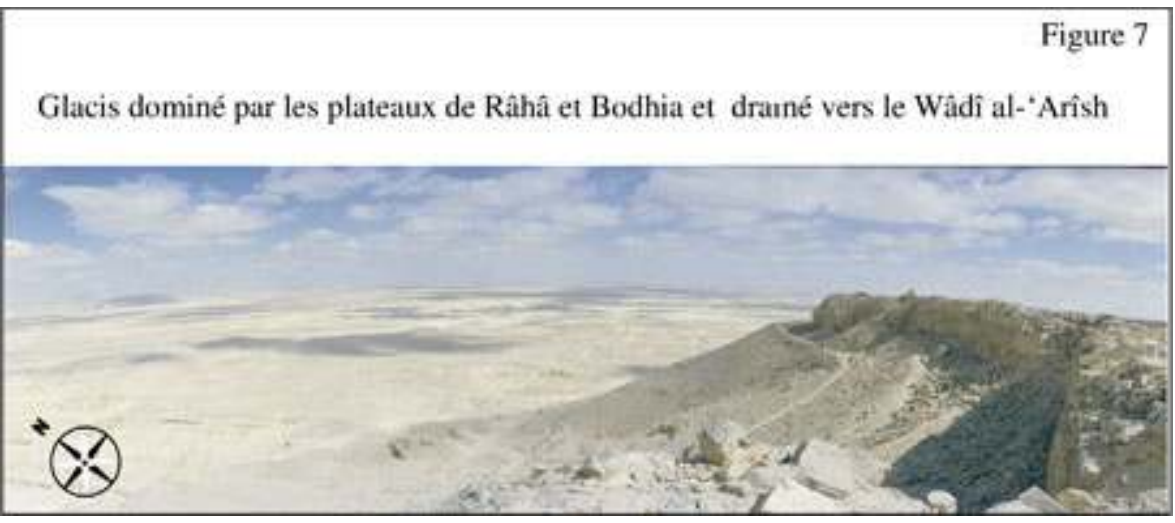

Bâti au sommet d'une butte témoin $(653 \mathrm{~m})$, la forteresse est érigée en position de bastion avancé. Véritable tours de guet du massif de Râhâ, elle contrôle le col entre le Jabal Gidi et le Gegel Somar situé directement au Sud. Ce col relie non seulement le golfe de Suez à la partie orientale de la Péninsule mais offre un accès à la Méditerranée par la plaine du Wâdî al-'Arîsh. A la fois large et peu dénivelé, il offre un chemin relativement confortable eu égard à la barrière massive que constitue le Jabal du Tih situé plus au sud. 

défensive et le commandement que ce soit en direction de Suez ou vers la large plaine d'épandage du Wâdî al-'Arîsh en direction du golfe d'Aqaba (Figure 8).

Figure 8

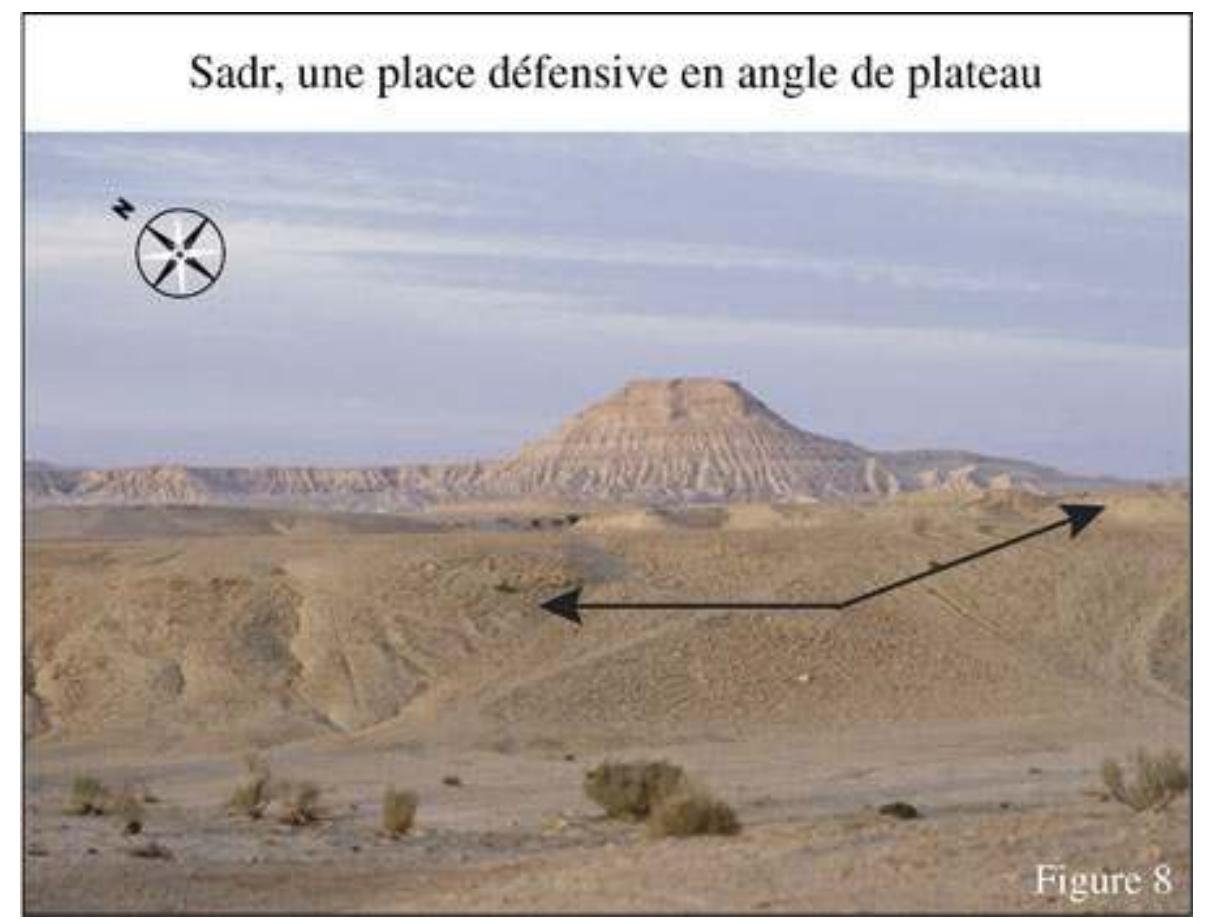

\section{La forteresse de Sadr ou le contrôle de la route de l'eau}

Une place défensive exige un approvisionnement en eau pour les 700 cavaliers accompagnants Saladin et un stockage suffisant pour pouvoir tenir et faire face à d'éventuel assaillants. En sus du dispositif à proprement structural qui permet le contrôle de la route entre l'Egypte et la Syrie, Saladin a du gérer la question de l'eau. Cette deuxième contrainte pour l'implantation de la forteresse n'est pas des moindres dans un milieu hostile et aride.

L'accès à l'eau constitue à la fois un objet symbolique du pouvoir et une véritable arme de guerre. Elle rentre pleinement dans les stratégies militaires de Saladin. Il l'utilisera d'ailleurs sous toutes ses formes. Les troupes de Saladin vont isoler et encercler l'armée du roi croisé Gui de Lusignan sur les hauteurs des Cornes de Hattîn, les coupant ainsi de tout accès à l'eau. Après la bataille de Hattîn, le sultan tend une coupe remplit d'eau fraîche au roi, ce qui signifiait qui lui faisait grâce de la vie. La forteresse de Sadr à deux ou trois jours de marche du Caire vers l'est et autant de Ayla vers l'ouest a été construite comme une véritable halte sur la route du Sinaï centrale. Les caravanes marchandes comme les cavaliers de Saladin devaient pouvoir y trouver eau, nourriture terrestre et spirituelle dans une des régions les plus sèches au monde. Saladin a fait bâtir sur une butte à plus de $650 \mathrm{~m}$ dont le seul accès se fait par un étroit sentier extrêmement raide à flanc de rocher L'intérêt de la gestion de l'eau est renforcé par les 
découvertes des fouilles qui ont mises au jour la présence de hammam (Figure 9a) et d'immenses citernes (Figure $9 \mathrm{~b}$ ) sous les mosquées (le volume de la plus petite citerne a été estimé entre 130-170m3). et une succession de deux barrages-réservoirs construit sur le wâdî Kuhayla à moins de $100 \mathrm{~m}$ d'intervalle au nord de la forteresse.(figure 10 ).

Figure 9

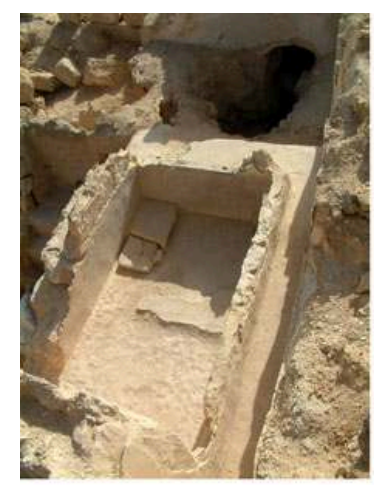

Bien que le hammam occupe une place majeure
dans Tarchitecture islamique, de parn sa double vocation, lieu de rituel de Pablution et lieu de détente, le soin apponée à sa construction dans w comtexte aride et austere pose question.

D'une surface de $40 \mathrm{~m}$ ? I le hammam se developpe sur decux niveaux et se divise en deux parties: les neur et celles reservies at a chavferies. Les pièces de services situées à proximite des grandes citernes de la forteresse, comprennent au rez-de-chaussíe une réserve de combustible et une chaufferie, et a létagge, une citerne déeuu
froide qui alimente par gravité les lavabos et los toiletes ainsi que la cuve destince à produire de enfilade dont ratmosphere esr plus ou moins petits corridors śquivalen de sus, qui per ar tent de réguler les échanges thermiques. la première salle non chauffiée est la salle de déshabillage, les deux suivantes sont chauffées a la fois par la vapeur provenant de la chaudiere productrice deau en ébullition et par un sol en hypocauste.
la salle du milieu est agrémentée de deux La salle du milicu est agrémentéé de deux
bassins en calcaire alimentés tant en eau chaud bassins en calcaine alimentes tant en eau chaude de dowe petits jouss en forme d'hoiles s six branches et de disques fermetes par des plaques de verre colorés.

Porte disccés au hammam et reservoir

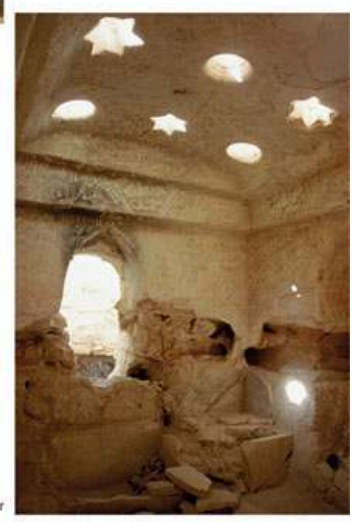


Figure 10
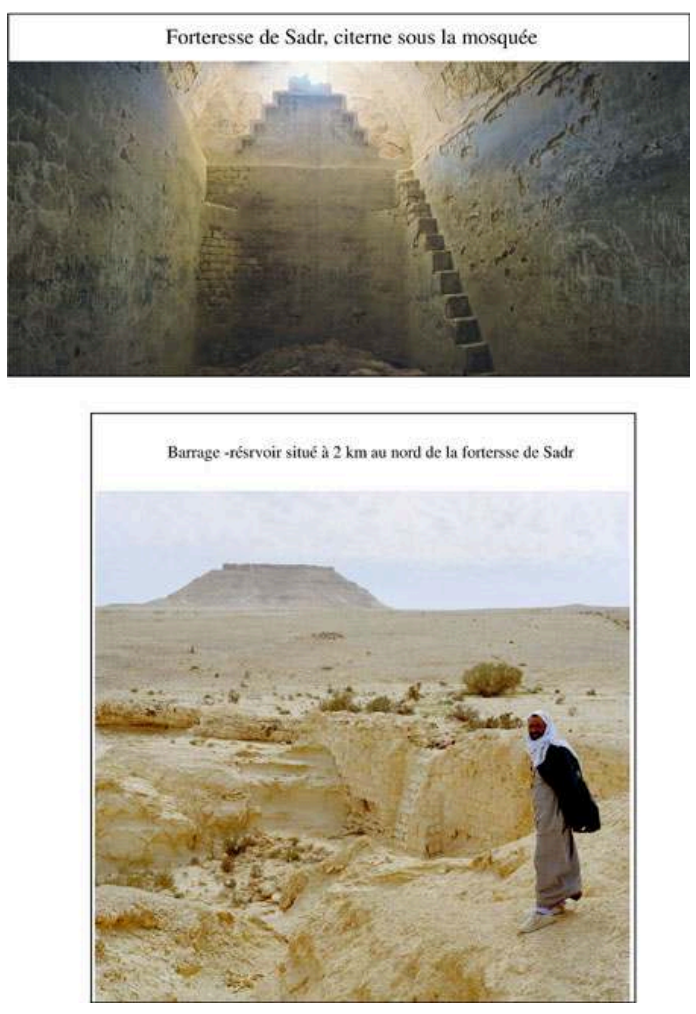

\section{Sadr au cœur d'une région hydrogéologique favorable}

La forteresse se situe dans une région où les précipitations annuelles sont inférieures à $50 \mathrm{~mm}$ par an et où les variations climatiques oscillent entre arides et hyper-arides. La limite entre la zone aride et hyper-aride est donnée par le rapport entre les précipitations et le potentiel d'évapotranspiration. Ce rapport est compris entre 0.03 et 0.2 pour les milieux arides et inférieur à 0.03 pour les zones hyperarides. Ce qui situe exception faite d'une bande littorale Nord et de la région montagneuse élevée du Tih, l'ensemble du Sinaï en zone hyper-aride comme le montre la carte des isohyètes du Sinai. (figure 11) 
Figure 11

Carte des précipitations du Sinai

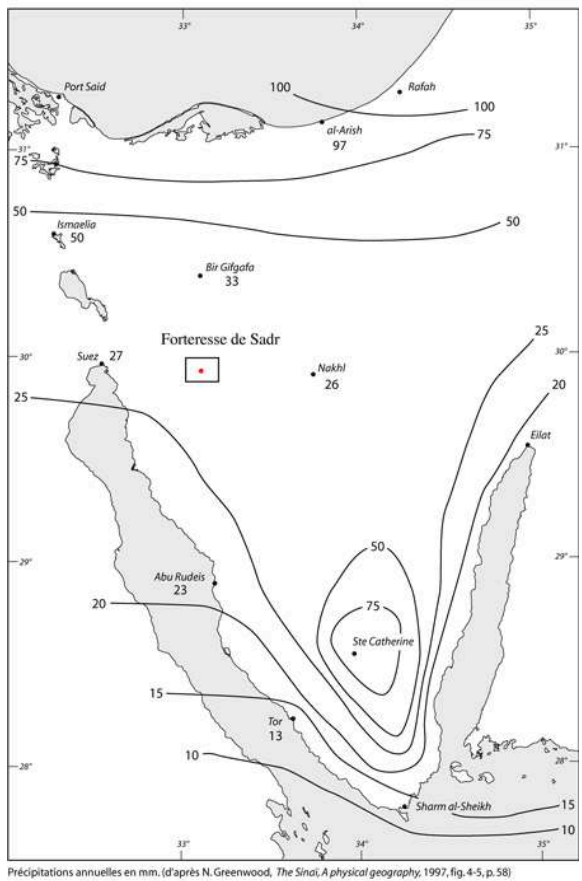

La forteresse est à quelques quatre kilomètres au nord de deux sources pérennes, de Ayn Tisar al-Malih et Ayn Sudr qui ont donné naissance à une petite oasis. Les sources correspondent à des résurgences d'eau douce, situées en limite amont du réseau hydrographique. Les faibles précipitations conjuguée à cette situation particulière, ne permettent pas à ces sources de se réalimenter. La pérennité des eaux est liée à la présence de nappes souterraines. L'ensemble des plateaux crétacé constitue en effet un bon aquifère où l'eau souterraine peut circuler aisément (figure 12). Ces plateaux occupent la quasi-totalité du désert de Tih et du Gabal Egma. Le secteur du Ayn Sudr constitue ainsi l'aval d'un vaste sous-bassin hydrogéologique.

D'autre part, un réseau de failles orientées nord-sud et est-ouest quadrille la région des sources favorisant l'infiltration des eaux et le transit des eaux entre différents aquifères.

37 Les eaux de sources seraient ainsi d'origine fossiles datant probablement, comme les formations qui accueillent l'aquifère, du Crétacé. 


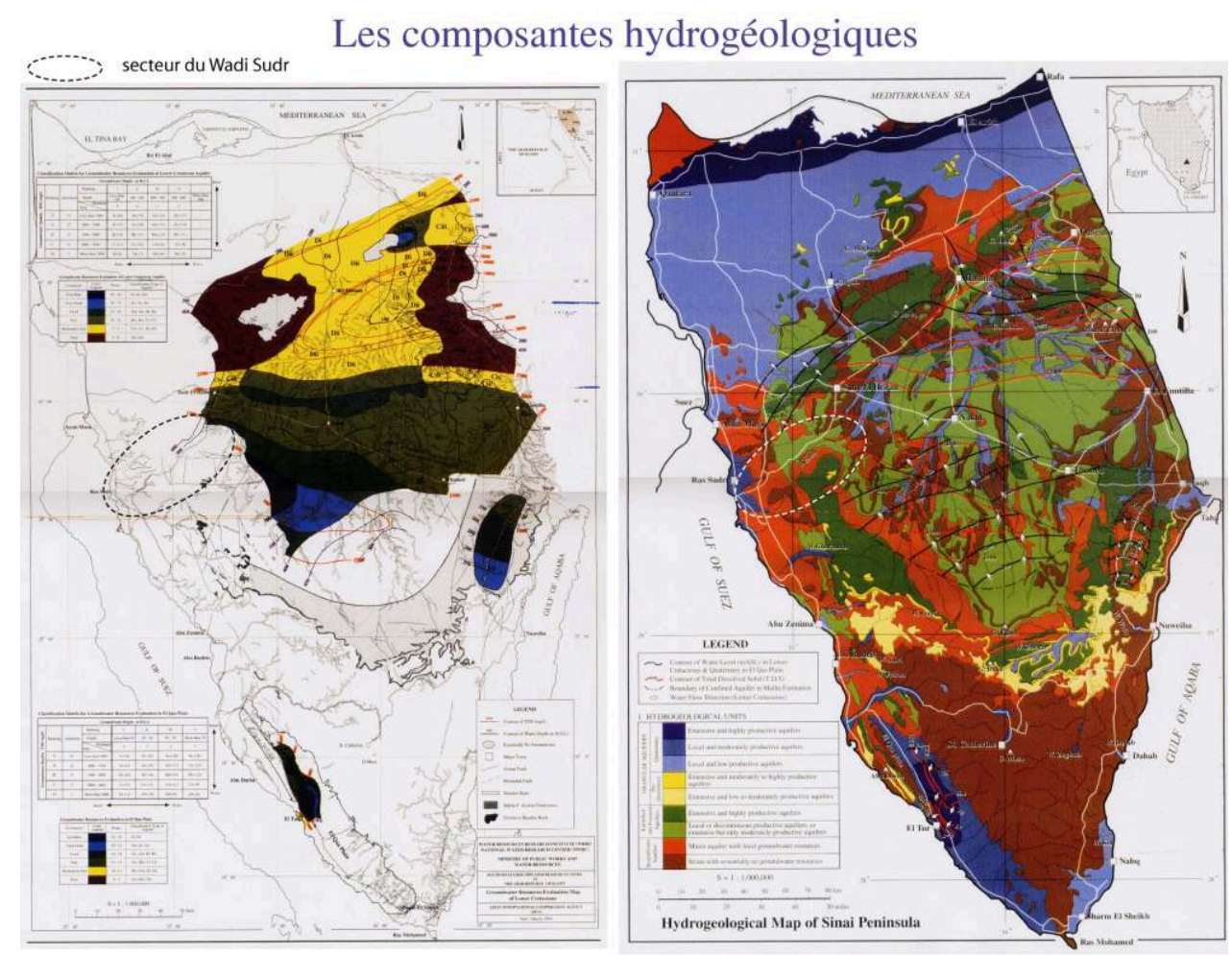

\section{Sadr, sur la ligne de partage des eaux.}

La butte se situe sur une position charnière. $28 \%$ du Sinaï est drainé par le bassin du Wâdî al-'Arîsh vers la Méditerranée. Trois systèmes principaux, wâdî Bruk, al-'Arîsh, et Aqaba drainent des centaines de petits wâdî qui convergent par un système complexe de chenaux vers la large vallée de sable du Wâdî al-'Arîsh entre Uyûn Mûsâ et Hamman Faraun (façade ouest de la péninsule) seuls trois systèmes de wâdîs (Lakata, Sudr et Wardan) maintiennent un écoulement vers le golfe de Suez.

Et précisément, la butte de Sadr se situe dans le secteur de partage des eaux de ces deux systèmes vers la Méditerranée par le bassin d'al-'Arîsh et vers Suez par le wâdî Sudr (figure 13).

40 L'intérêt de cette position est éminemment stratégique, elle offre une double possibilité d'alimentation en eau pour la forteresse.

\section{Sadr campe en angle du plateau de Râhâ offrant deux lignes pour de possibles résurgences d'eau}

41 La position avancée de la forteresse au sommet d'un angle formé par deux lignes de directions N.E:S.O et N.O./ S.E. a pu donner lieu à des résurgences d'eau douce qui ont pu se déplacer le long de ces lignes au fil du temps La construction du barrage à $2 \mathrm{~km}$ au nord de la forteresse laisse penser que ces lignes de sources ont fonctionné au côté de l'impluvium que constituent les versants du plateau de Râhâ.

Aussi est-il difficile de localiser avec précision la station originelle de Sadr. La source actuelle, située à 4 kilomètres au sud de la forteresse n'est probablement pas celle qui a 
fonctionné entre 1150 et 1250 ; elle n'a en tout cas aujourd'hui qu'un débit très faible, incapable de pourvoir aux besoins de la forteresse et encore moins d'alimenter et de remplir barrages et citernes (figure 14).

Figures 13 et 14

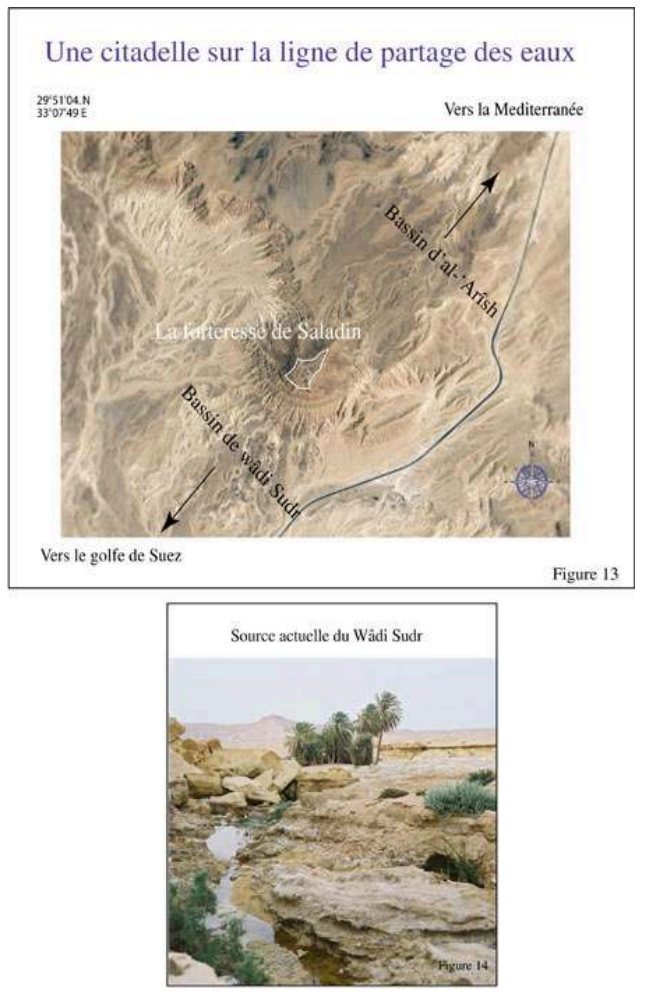

Le régime des pluies est strictement méditerranéen, c'est le passage les dépressions hivernales qui se situent de décembre à février, qui assure les précipitations. L'humidité relative décroît vers le sud.

Le wâdî Kuhayla affluent du Wâdî al-'Arîsh n'est alimenté que de façon exceptionnelle par les violents orages qui peuvent s'abattre sur ce secteur, et leur occurrence n'est que d'une à deux fois par an; Or, même lors de ces crises pluviométriques, il n'est pas sûr que les deux barrages auraient pu être rempli.

45 Il est fort probable que la forteresse de Sadr a bénéficiée de conditions climatiques plus favorables que l'actuel. Les précipitations auraient pu été plus abondantes entre 1175 et le milieu du XIII è siècle.

L'implantation des barrages au nord de la forteresse pourrait répondre à des conditions locales.

\section{Sadr, une butte face à la circulation générale}

Il est probable qu'à l'époque de l'occupation du site une source se trouvait à l'amont du wâdî Kuhayla qui aujourd'hui ne fonctionnerait plus. D'autre part, les versants nord des reliefs auraient pu être plus humides.

Les précipitations sont en effet apportées par des vents de Nord. La butte de Sadr en position avancée par rapport au plateau présente un versant large et raide au vent. Or ce relief pourrait être à l'origine d'un effet de foehn. Le courant aérien qui rencontre la 
butte ne pouvant la contourner va s'élever, c'est-ce qu'on appelle l'ascendance orographique. L'air en montant subit une détente et se refroidit. Si l'air atteint le point de condensation, des précipitations se déclenchent. Les barrages situés au nord de la forteresse auraient pu être construits pour recueillir et stocker ses eaux providentielles.

Dans le Sinaï, les barrages-réservoirs sont le plus souvent accompagnés de constructions de pierre qui officient comme de véritables chenaux latéraux pour dériver et amener davantage d'eau au barrage. Or, nous ne retrouvons ce système sur aucun des deux barrages. L'origine de l'alimentation a donc du être suffisante, et plaident en faveur d'une alimentation autonome. Les barrages pourraient être les témoins $d u$ fonctionnement relativement régulier de ce système de foehn et étayer l'idée d'un optimum climatique.

\section{La vie de la forteresse aurait-elle été réglée par les oscillations climatiques?}

Des variations climatiques plus ou moins importantes se sont succédées dans l'histoire, y compris à l'échelle historique. Les aléas constituent peut-être une clef pour comprendre d'une part l'édification de barrages, citernes et de hammams et d'autre part l'abandon de la forteresse vers le milieu du XIIIè siècle. Le développement de la forteresse tend à être synchrone avec la période de l'optimum climatique médiéval.

51 Le réchauffement médiéval semble s'étendre de 1150 à 1250, les températures se sont élevées de $+1^{\circ} \mathrm{C}$. Ce réchauffement a permis l'installation de la vie au Groenland, la culture de la vigne jusqu'en Ecosse vers $400 \mathrm{~m}$ d'altitude. Nous assistons en Europe à une période d'accroissement démographique et de développement technique. Il est donc fort probablement qu'à cette période le secteur de Sadr ait été mieux alimenté par les eaux de pluie.

52 Les analyses scientifiques comme les sources historiques convergent vers cette hypothèse.

\section{Les enregistrements scientifiques}

53 E. Le Roy Ladurie ${ }^{8}$ dans l'analyse des faits historiques nous renseigne sur deux Petits âges de glace pour cette période.

Un premier vers 900AD et un second vers 1250-1300, «bref mais intense ", ce qui induit un Moyen Age plus chaud et plus humide entre ces deux épisodes.

Il note une dégradation rapide du climat vers 1250 . Plusieurs indices vont dans ce sens : les étés sont plus frais, et les récoltes de céréales diminuent considérablement sur l'occident, les tempêtes ravagent les ports hollandais.

W. S.Broecker ${ }^{9}$ mentionne dans ses reconstitutions des températures à partir des analyses dans les forages dans la glace du Groenland et des évènements historiques comme la destruction du bisse (canal d'irrigation pour l'agriculture, en pays de faible précipitation) issu du glacier d'Aletsch une large extension du PAG vers 1300 . Ce dernier a dû par conséquent se mettre en place beaucoup plus tôt. 
D. Dahl-Jenssen ${ }^{10}$ enregistre également dans deux forages profonds (GRIP et Dye 3) un optimum climatique avant 1300 . Ces forages retranscrivent des signaux à basse fréquence, c'est-à-dire ceux d'évènements majeures dans leur grande amplitude. Il convient donc de faire débuter le PAG plus précocement.

\section{Les sources historiques}

Plusieurs sources historiques mentionnent d'ailleurs Sadr comme un lieu fort bien alimenté en eau.

L'historien copte Abû l-Makârim ${ }^{11}$ (vers 1170-1200) en dressant la liste des points d'eau du Sinaï, mentionne Sadr parmi les mieux alimentés.

Al-Jazarî12 note que dans le désert de Tîh à proximité du Jabal Hasan, coule une source d'eau appelée Sadr.

Les secrétaires de Saladin qui séjournent dans la forteresse la décrivent comme une station où l'eau est abondante au regard des paysages arides du Sinai central.

L'optimum climatique médiéval aurait ainsi permis à la forteresse de fonctionner et d'alimenter en eau pèlerins et cavaliers de Saladin. La situation remarquable de la forteresse, située à la fois sur la ligne de partage des eaux entre méditerranée et golfe de Suez et à l'angle du plateau de Râhâ, se trouve au centre d'un véritable système d'alimentation naturelle en eau douce.

3 La route de Saladin traverse deux bassins versants, le Wâdî al-'Arîsh et le Wâdî al-'Sudr. Ces deux bassins, de tailles différentes fonctionnent à des vitesses différentes et offrent donc une alimentation en eau décalée dans le temps. Le glacis qui s'étire en contre bas de la butte de Sadr a permit l'installation d'un double barrage-réservoir situé au nord de la forteresse. Ces réservoirs ont probablement pu bénéficier de conditions d'alimentation très favorables puisque situé sous le flanc nord de la butte.

64 Les raisons politiques, militaires, économiques et géographiques se sont croisées sur la route du Sinaï intérieure;

route de Saladin et de ses cavaliers dans leurs offensives contre les croisés, route de la Terre Sainte, route caravanière, route des liaisons commerciales entre l'Afrique, l'Inde et l'Extrême-Orient via la Mer Rouge, route naturelle de l'eau, et enfin routes scientifiques.

6 La forteresse de Sadr, à mi chemin entre le Caire et Ayla, a constitué un pivot indispensable quand les Etats croisés contrôlaient la route côtière.

Elle fût implantée sur une haute butte témoin en position de place forte, dans un secteur où l'approvisionnement en eau bénéficiait de plusieurs sources.

\section{En conclusion}

68 L'histoire se superpose pour un temps à la géographie. De la stratégie militaire à une réponse guidée par le milieu, quelle est la part de chacun dans la construction de la forteresse de Sadr?

Dans un contexte hostile, austère et aride, Sadr représente un modèle d'adaptation, d'anthropisation d'un espace en milieu extrême. Il y a eu dans l'histoire de la forteresse 
un important déterminisme physique et climatique mais les choix ont été déterminés et portés par le culturel et les logiques historiques.

Cette guerre de l'eau s'est appuyée à la fois sur une stratégie militaire mais aussi sur une réponse adaptative immédiate au désert qui a nécessité des choix techniques. En terme d'adaptation, la quête de l'eau a entraîné une gestion spécifique et de l'espace et de ces contraintes.

Echappons ainsi à la question du déterminisme physique qui se réveille doucement sous la brise du désert.

\section{NOTES}

1. X. de Planhol. 1975 L’Egypte.Géographie régionale I. Encyclopédie de la pléiade. p,1731.

2. J.M. Mouton Saladin, le sultan chevalier. Découverte Gallimard, Paris, 2001

3. Amiral H. Labrousse. Récits de la mer Rouge et de l'Océan Indien. Etudes d'Histoire maritime 1992,volume 10.

4. R. Grousset, Histoire des Croisades et du Royaume franc de Jérusalem, 3 Vol. Paris, 1935.

5. J.P. Prawer, Histoire du royaume latin de Jérusalem, tome I, traduit de l'hébreu par G. Nahon, Paris, 1969

6. Schlumberger, Renaud de Châtillon, Prince d'Antioche, Paris, 1898

7. Schlumberger, p. 274

8. E. Le Roy Ladurie.. (1983) Histoire du climat depuis l'an Mil., Flammarion, vol I et II.

9. W. S.Broecker (2001)_ Was the Medieval warm Period Global ? Science, 291, 1497-99

10. D. Dahl-Jenssen et al.(1998)_Past temperatures directly from the Greenland Ice sheet, Science, Science, 282, 268-271.

11. Abû l-Makârim, Ta'rîkh al-kanâ'is wa l-adyûrâ', III,p.1

12. Al-Jazarî_( 1964) Durar al-Fawâid, Le Caire, p. 488

\section{RÉSUMÉS}

La forteresse de Sadr, située dans le secteur le plus aride du Sinaï, a été construite par le sultan Saladin dans le contexte historique des croisades. Ce site archéologique très particulier a été lu à la fois comme un élément physique dans l'histoire du paysage et comme une inscription de l'histoire dans le paysage.

At the heart of the Sinai desert lies the Sadr fortress, today called Qal-at al-Gindi, is the most authentic archeological legacy left in the Middle East by the famous Kurdish sovereign Saladin (1174-1193). This military edifice was built in the context of the crusades. The organization of the east-west crossings of the Sinai was in fact going through a major change in the twelfth century. The low coastal route along the Mediterranean provided a quick and easy way for Muslim 
caravans traveling between Egypt and Syria. The littoral plain of Gifar offered a dune belt that was relatively flat compared to the mountains of central Sinai. This road, a main route for centuries, came under partial control of the Frankish states in 1099 with the creation of the Latin Kingdom of Jerusalem.

The Latin hold became a more impassible obstacle than the physical barriers of the Sinai. Saladin therefore set up an inner route that made the journey between Africa and Muslim Asia slower and harder but safer. Little by little, control over physical constraints began to work against the crusaders. The location of the Sadr fortress appears to have met two major geographic criteria. Firstly, construction on a high butte gave the fortress a strong defensive position with a commanding view of the lower areas, which were the only way an army could approach. Secondly, the fortress's location provided exceptional access to fresh water.

The Sadr fortress in central Sinai, in the Wâdi-al-Sudr valley eighty kilometers from the Gulf of Suez, was built on and for the central Sinai route. Midway between Cairo and the Ayla fortress, it constituted a vital stopover for Saladin in an extremely harsh and arid region.

\section{INDEX}

Keywords : Sinai, geography, history, Sadr fortress, Saladin

Mots-clés : Sinaï, géographie, histoire, forteresse de Sadr, Saladin

\section{AUTEURS}

\section{CORINNE FEİSS-JEHEL}

corinne.feiss@cnrs-bellevue.fr Maître de conférences (Géographie, Sciences de la vie et de la terre) à l'EPHE.

\section{JEAN-MICHEL MOUTON}

jm.mouton@wanadoo.fr, Directeur d'études (Sciences historiques et philologiques à l'EPHE.

\section{CLAUDINE PIATON}

Architecte des bâtiments de France.

PIERRE-JÉRÔME JEHEL

Photographe Ecole des Gobelins.

JEANNINE LE RHUN

Maître de Conférences (Géographie, Sciences de la vie et de la terre) à l'EPHE. 